\title{
Assistive Technology for Disabled Persons
}

\author{
Aslam Muhammad ${ }^{1}$, Warda Ahmad ${ }^{2}$, Tooba Maryam ${ }^{3}$, Sidra Anwar ${ }^{4}$ \\ Department of CS \&, U. E. T., Lahore, Pakistan \\ (balochwarda@gmail.com, tooba_islamic@yahoo.com, sidra.anwar72@yahoo.com) \\ Martinez Enriquez A. M. \\ Center of Investigation and Advanced Studies (CINVESTAV), D.F. Mexico (ammartin@ cinvestav.mx $)$
}

\begin{abstract}
Assistive technology aims to serve the disabled people, who are unable to do their daily routines with ease. Despite the emphasis on mechanics and the rapid proliferation of modern devices, little is known about the specific uses of such gadgets introduced now-a-days. Guardians of fully/partially sighted and handicapped persons remain indecisive in making selection of required tools. Thus, the purpose of this work is to help people in choosing the best suited widgets for them. We conduct a parameterized review and a systematic analysis of Windows and Linux based assistive artificial supporting agents. This study is carried out to show the currently available assistive crafts, their working, effectiveness, performance, and cost. On this basis of the review, recommendations are given to overcome their shortcomings.
\end{abstract}

Keywords: Assistive technology, disabled persons, user interface, disability.

\section{INTRODUCTION}

Around 15 per cent of the world's population or estimated 1 billion people live with disabilities. Approximately, 90\% disabled people live in developing countries [1]. According to United Nations Children's Fund (UNICEF), there are an estimated 150 million children with disabilities in the world [2]. People who are blind cannot read instructions presented only in a visual format; people who are deaf cannot understand content that is presented only aurally and people with mobility and dexterity impairments might not be able to use arms or fingers to interact with their computer using standard keyboard or mouse. So, disabled persons cannot access the technology for their educational needs and its communication in educational settings [3]. To access the modern gadgets they need assistive technology devices.

Assistive Technology (AT) is an any item, piece of equipment, or product system, whether acquired commercially off the shelf, modified, or customized, that is used to increase, maintain, or improve functional capabilities of individuals with disabilities[4]. Examples of assistive technology include wheelchairs, electronic communication systems, computer adaptations, and thousands of other commercially available devices. During the last two decades, great attention has been given to the development and evaluation of technological devices to assist disabled persons [5].

A tremendous variety of assistive tools are available today worldwide, providing the opportunity for nearly all people including physically and mentally challenged persons to access Information Technology (IT) - e.g., Braille display, Screen readers, voice recognition programs, speech synthesizer, screen magnifier, teletypewriters conversion modem, etc. [30]. These gadgets can include hardware, software, and peripherals that assist people with disabilities.

Almost all the famous operating systems like Windows, Linux, and Solaris, etc. have some build-in accessibility features for disabled people e.g. Narrator, Vinux and complete mouseless access to the desktop respectively. Along with these build-in features they also provide support to wide range of assistive technologies available commercially and for free.

Unfortunately, many teachers and special educators don't realize that how assistive technology can benefit disables persons. The people who have some realization have a question that which assistive technology device or software is considered for persons with a disability. Evaluating the technical needs of individual with disabilities, parents or instructors are unable to identify the appropriate assistive technology hardware or software that increase the functional capability of disabled persons in home, school, or society?

In this paper, we address issues regarding selection of Microsoft Windows and Linux based assistive technology for physically disabled persons. We choose to review Microsoft Windows and Linux based assistive technology hardware or software because Microsoft Windows and Linux are one of the widely used operating system and it provides wide range of interfaces for disabled persons [6][30]. These are also compatible with a wide variety of assistive technology products such as screen readers, magnifiers, and specialty hardware that meet the needs of computer users with all types of physical impairments. We carried out a systematic review on selected Microsoft Windows and Linux based assistive technology hardware or software.

Section II gives an overview of related work done in this field. Section III describes the set of parameters that are selected for the analysis of assistive technology and parameterized based analysis of Windows based assistive technology products is also presented in this section. While parameterized based analysis of Linux based assistive technology tools are presented in Section IV. Section V discusses the shortcomings and recommendations in these widgets. Section VI concludes the paper and gives future perspectives. 
Table 1: Assistive technology products

\begin{tabular}{|c|c|c|c|c|}
\hline Vendor Name & Product Type & Product Name & Supportive Disease & $\begin{array}{l}\text { Hardware } \\
\text { or } \\
\text { Software }\end{array}$ \\
\hline Origin Instruments & Alternative Input & Head Mouse [12] & Mobility Impairment & Hardware \\
\hline Fentek Industeries & Foot Mouse & Foot Mouse [13] & Mobility Impairment & Hardware \\
\hline $\begin{array}{l}\text { Boundless Assistive } \\
\text { Technology }\end{array}$ & On-screen keyboard & $\begin{array}{l}\text { SofType English on } \\
\text { screen keyboard [14] }\end{array}$ & Mobility Impairment & Software \\
\hline Icommunicator & $\begin{array}{l}\text { Voice synthesizer/ } \\
\text { Speech to text }\end{array}$ & Icommunicator [15] & Deafness & Hardware \\
\hline Freedom Scientific & Screen readers & $\begin{array}{l}\text { Job Access with Speech } \\
{[16]}\end{array}$ & Blindness & Software \\
\hline NV Access & Screen reader & $\begin{array}{l}\text { Non Visual Desktop } \\
\text { Application [18] }\end{array}$ & Blindness & Software \\
\hline Freedom Scientific & Scanning and reading & $\begin{array}{l}\text { Open Book and Pearl } \\
{[19]}\end{array}$ & Blindness & Hardware \\
\hline Vinux project & Linux distribution & Vinux [27] & Blindness & Software \\
\hline Gnome & Screen reader & Orca [28] & Blindness & Software \\
\hline Emacspeak Inc & Screen reader & Emacspeek [26] & Blindness & Software \\
\hline
\end{tabular}

\section{RELATED WORK}

In the past, much work has been done in the field of assistive technology. A lot of literature review papers are published during last two decades.

A study conducted by Sandra Alper and Sahoby Raharinirina shows a comprehensive review and a systematic analysis of published reports of assistive technology and skill gaining of persons with disabilities. Uses of assistive technology, its benefits and obstacles, are reviewed. The results provide indications why technology is often abandoned. Implications for practitioners and researchers are discussed in it [8].

A literature review was conducted with the objective to evaluate the usability of assistive technology to the people with dementia. Fifty four relevant papers were considered in detail during this review. Studies have used technology for prompting and reminding as loss of time and forgetfulness are major problems; for monitoring daily activities in a sensor enriched environment and utilized location aware technologies to provide information to enhance safety. Technology also supports a range of therapies to alleviate symptoms. Therapies include the delivery of music and familial pictures for reminiscing, the use of light therapy to enhance wellbeing and the provision of mental tasks to stimulate the brain and maintain activity levels [9].

Another study was conducted by Hoppestad BS .This study provides a review of the existing literature regarding computer access for persons with multiple disabilities using assistive technology (AT). Subjectively, It is assumed that disabled persons are underserved by the AT community, and the causalities behind this perception were explored [10].

Another exploratory study summarizes the results related to the use of technology in helping students with learning disabilities. The main objectives of this study are to detect the specific technology suggestions found in the literature, to find out the problems related to using these suggestions in the transition to postsecondary education and to provide suggestions for planning for the transition to postsecondary education [11].
Until now, just a few reviews had been conducted. They differ from the review presented here in several ways: they were restricted to literature, they didn't consider operating system, and they considered only one disability/impairment. There is an absence of systematic review of assistive technology hardware or software based on Microsoft Windows and Linux operating system for disabled persons.

\section{PARAMETERIZED ANALYSIS OF WINDOWS BASED ASSISTIVE TECHNOLOGY PRODUCTS}

Our review focuses on the leading operating systems for laptops and personal computers, Microsoft Windows and Linux. The analysis is carried out using Microsoft Accessibility website and Linux Accessibility HOWTO website for Microsoft Windows and Linux based assistive technology products assistive technology products respectively [7] [29]. We focus exclusively on the items related to blindness, deafness, and mobility impairment.

In first step, we visit the vendors/creators of different assistive technology products supported by Microsoft Windows and Linux. Then, analyze the products related to the blindness, deafness, and mobility impairment. Selected products are included in Table 1 . We consider all the available information given by both the accessibility websites and the vendor website and collect the information for all selected products. We also consider some online comparison and review articles related to these products.

A systematic review is carried out on the basis of set parameters such as performance, usefulness, user-friendly, local languages support, functionality, portability and acquisition cost.

Performance is a parameter that is used to review an assistive technology in terms of responsiveness, reliability and resource usage. Usefulness means reviewing an assistive technology product that how much it is helpful to disable person. User-friendly is a parameter that is used to review the ease of usage of assistive technology product. Local language support is also reviewed to help the disable people who don't understand English language and needed the support of their local language. 
Functionality is used to review that an assistive technology product provides set the functionalities it is made for or not. Portability is also a very important parameter specially reviewing an assistive technology for mobility impaired persons. Portability is used to review a product that a disabled person can carry it easily with him or not. Cost is an important factor in developing countries. Cost is also considered while reviewing these products [23].

Next in this section, an analysis is carried out on assistive technology products based on Microsoft Windows mentioned in Table. 1.

\section{A. Non Visual Desktop Access (NVDA)}

NVDA is a free and open source screen reader which allows blind and vision impaired people to use computers. It helps blind people by reading the text on the screen in a computerized voice. The text is read by moving the cursor to the relevant area of text with the keyboard arrows or a mouse [18]. It is compatible with Microsoft Windows. It also supports refreshable Braille displays [20]. It has no additional hardware requirements and needs only 50MB of disk space.

It is helpful in education and employment for many blind people. Its general features are browsing the Web, word processing, built in speech synthesizer, computer management, telecommunication and automatic reading the text under the mouse [22]. But it doesn't have low vision features such as reverse contrast and magnifier.

One exciting fact about NVDA is its support for multiple local languages. Besides English, its interface has been translated into more than 36 languages. It has a built- in speech synthesizer which can read contents in more than 80 languages. It is easy to use and understand. It can be installed on the computer by using easy to use talking installer. It also has a portable version. So, it can also be run from USB stick without the need for installation. Normally screen readers are pricy. Blind people can't afford them. NVDA is a free of cost software.

\section{B. Job Access with Speech (JAWS)}

JAWS is a powerful and most popular screen reader, which is developed by freedom scientific for computer users whose vision loss prevents them from seeing screen content or navigating with a mouse [16]. It enables blind and visually impaired people to gain access to information on their computer. It helps to provide access to software applications and the Internet. It also outputs to refreshable Braille displays [21].

It is compatible with Windows OS. Additional Hardware requirements are Windows compatible sound card and a display adapter. JAWS is simple and easy to use software. It is an easy program to load onto a computer. It is installed by using a talking installer. Its portable version is also available. It also supports upto30 different languages. Its documentation is also available in multiple languages for help. It is expensive software. Its professional version costs $\$ 1,095$ and standard version costs $\$ 895$.

\section{OpenBook and Pearl}

OpenBook and Pearl is scanning and reading system. It converts printed documents or graphic-based text into an electronic text format on computer, using high-quality speech and the latest optical character recognition technology. It helps blind and visually impaired people to access the printed and electronic materials. It has two parts: OpenBook and Pearl. Pearl is a portable reading camera used in combination with software such as OpenBook 9 or later[19].

The recommended system requirements for OpenBook are all 32 bit versions of Windows XP, all 32 and 64 bit versions of Windows Vista and Windows 7, $500 \mathrm{MHZ}$ Intel Pentium processor or faster, $256 \mathrm{MB}$ RAM and 1.2 GB of hard disk space. Additional requirements are sound card, video card, acquisition device such as Pearl camera and 104 keys keyboard.

OpenBook is simple and easy to use software. Its interface is simple and easy to understand. The user guide of OpenBook and Pearl is available online for help. It also supports multiple languages. It converts printed text to human like speech in many languages.

Another feature of this system is portability of the Pearl camera. It runs off a single USB port, no extra power supply or battery is required. The Pearls sets up in seconds and also folds up into a compact package and zips into its protective neoprene case. It is very costly system. OpenBook and Pearl costs up to $\$ 1,790$.

\section{D. iCommunicator}

The iCommunicator program helps persons who are deaf, hard-of-hearing, or those with other communication challenges achieve two-way communication. It is an interactive solution to communication accessibility needs in educational, workplace, and public access venues. It is also a teaching tool that helps improve literacy skills. It converts speech into multiple streams of data for simultaneous presentation of text, video-sign language, and computer- generated speech. The iCommunicator software works with Dragon Naturally-Speaking speech recognition software allowing you to train your computer to recognize voice and speech patterns. The program stores these voice files for immediate access [15].

In the era of multi-processor computing, iCommunicator can easily be installed and run on Pentium III. It is very useful for the people who are deaf or hard of hearing. It translates Speech to Text, Speech/Text to Video SignLanguage and Speech/Text to Computer Generated Voice. It is not intended as a replacement for sign-language interpreters, but as an alternative when an interpreter is not available: classrooms, daily communication, and emergency situations.

iCommunicator is easy to install and easy to use. Its interface is very simple and easy to understand. The user guide provided with the software is very simple and provides to the point guidance to the disable persons. But, the current version of iCommunicator doesn't support any language other than English. It provides the promised set of functionalities, but on a very high cost. It is very costly and supports only LightSPEED 360 FM system and TK-250 noise-canceling microphone provided by iCommunicator itself. So its maintenance is also very costly.

\section{E. $\quad$ SofType English on Screen Keyboard}

SofType is a software utility which replaces the functionality of a standard keyboard with a full featured on- 
Table 2: Parameterized comparison of Windows based assistive technology products

\begin{tabular}{|l|l|l|l|l|l|l|l|}
\hline \multicolumn{1}{|c|}{ Tools } & NVDA & JAWS & $\begin{array}{l}\text { OpenBook } \\
\text { and Pearl }\end{array}$ & iCommunicator & $\begin{array}{l}\text { Soft } \\
\text { Type }\end{array}$ & $\begin{array}{l}\text { Head } \\
\text { Mouse } \\
\text { Extreme }\end{array}$ & $\begin{array}{l}\text { Footime } \\
\text { Foot } \\
\text { Mouse }\end{array}$ \\
\hline Performance & High & Medium & High & High & High & High & High \\
\hline Usefulness & Medium & Medium & Medium & Medium & High & Medium & Medium \\
\hline User friendly & High & High & High & High & High & High & High \\
\hline $\begin{array}{l}\text { Local language } \\
\text { support }\end{array}$ & Yes & Yes & Yes & No & Yes & Yes & Yes \\
\hline Functionality & High & High & Medium & High & High & Medium & High \\
\hline Portability & Yes & Yes & Yes & Yes & Yes & Yes & Yes \\
\hline Cost & Free & Expensive & Expensive & Expensive & Affordable & Expensive & Affordable \\
\hline
\end{tabular}

-screen keyboard. It can be accessed using a mouse or mouse emulator such as the Head Mouse Extreme. It works by generating an image of a keyboard on the computer screen. When a key is selected, the character represented by that key is sent to the active Windows application [14].

It is easy to install on Windows OS and doesn't require any specific resources. It is designed to work in place of a keyboard to make computers accessible for individuals with physical limitations due to Cerebral Palsy, Parkinson's disease, paralysis, Muscular Dystrophy, Multiple Sclerosis, head injury or loss of a limb. It is easy to install and easy to use. Its interface is very simple and easy to understand. The user guide provided with the software is very simple and provides to the point guidance to the disable persons. Along with all this its 60 days trial version is freely available to test its functionality. Current version of SofType supports all international languages supported by Windows.

SofType has all promised features like AutoClick and Dragger, Predictive Word Completion, twelve different layout supports, Macro and Abbreviation Expansion, etc. Its cost is also affordable as compare to its features and other such software available in market.

\section{F. $\quad$ Footime Foot Mouse}

Foot Mouse is helpful for those people who have or want to avoid carpal tunnel syndrome and mouse generated Repetitive Stress Injury associated with desktop mice. It eliminates stress on the hand-wrist area by moving mouse control to the feet. It prevents wrist, elbow, and shoulder positioning before chronic pain develops. By using this person can get rid of wasteful, repetitive "keyboard-tomouse" hand movements increasing productivity. Another advantage of Footime foot mouse is that a person can have complete control of the cursor without having to take his/her hands off the keyboard or his/her eyes off the monitor.

It is compatible with Microsoft Windows. It is powered by user friendly Neuratron ${ }^{\mathrm{TM}}$ software. It works independently without affecting other hand mouse or input devices connected to the same computer at the same time. Its cost is $\$ 149.9$ which is not so high for those people who can't speak, listen, and they just have their foot and eyes working [13].

\section{G. HeadMouse Extreme}

HeadMouse Extreme is helpful for those people who cannot use or have limited use of their hands when controlling a computer. It translates natural movements of a user's head into directly proportional movements of the computer mouse pointer, so as the user moves their head the mouse pointer on the screen also moves. It has a tiny disposal target that is worn by the user on his head and a wireless optical sensor which tracks that tiny disposable target that is worn by the user. When, user head moves wireless optical sensor track user head location. In this way, it works just like a computer mouse [12].

It is also used with an on-screen keyboard such as SofType $^{\mathrm{TM}}$ or KeyStrokes ${ }^{\mathrm{TM}}$, it provides head-controlled access to all of the full range of functions for both keyboard and mouse. It is portable because its size is very small i.e. pocket size. It is compatible with Windows and Linux. It does not require any extra software for installation. It works with already present mouse drivers.

Usually computers can take 60 seconds to recognize it when it is first time plug in to it through a USB. It is very easy to install just plug in the USB and that's it. It does not consume any extra power because it uses the power of computer system. Its cost is not fixed i.e. varies according to the vender.

Table 2 compares Windows based assistive technology products on set of parameters that have already been defined. The criteria for these parameters are defined here.

Performance is high when the product has small response time, highly reliable and low resource usage. The performance is medium when the product is not fulfilling one of the above merits and low when the product fails to meet none of them. Usefulness is high when the product is helpful. Medium when it misses some of the features and low when it fails to fulfill its purpose. The product is highly user friendly when it is easy to understand, learn and use, medium when it's GUI is little tricky and low when it's complicated for its users.

Local language support of the product is when it supports any language other than by default one. Product's functionality is high when it meets all the stated or offered set of functionalities, medium when it misses some of them and low when it fails to meet its purpose. A product is portable when it is easy to move from one environment to other without any effort. The product is expensive when its cost is more than $\$ 500$. Affordable when it is between $\$ 100$ \$499. And cheap when it is less than $\$ 100$.

\section{PARAMETERIZED ANALYSIS OF LINUX BASED ASSISTIVE TECHNOLOGY PRODUCTS}

In this section, an analysis is carried out on some assistive technology products based on Microsoft Windows mentioned in Table 1. 


\section{A. $\quad$ Orca}

Orca is a free and open-source screen reader that allows users to access the graphical desktop through various combinations of Braille, speech and/or magnification. It is also known as a scriptable screen reader because it provides customized behavior based on the application with which it is working. It easily runs on distributions based including, Ubuntu, Debian, Fedora, Solaris Express and so on. It works with the applications that support the Assistive Technology Service Provider Interface (AT-SPI). It has also been translated into many languages [28]. It provides the promised set of functionalities. It is easy to install but it is not so much user friendly. As it is open source software, so it is free of cost.

\section{B. Vinux}

Vinux is a Linux or Ubuntu derived distribution which has been specially designed for visually impaired users. By default it provides users two screen readers, two screen magnifiers, global font-size and colour changing facilities. When you boot the live Vinux image, user is greeted by the Orca screen reader enabling user to navigate the graphical Gnome desktop using keyboard commands. Finally, Brailly provides Grade 1 and 2 Braille output via Orca [27].

The performance of the Vinux is completely based on Linux's performance because it ultimately based on Ubuntu. It is very useful for the people who are blind and partially sighted as it contains a set of applications in one distribution. Vinux is easy to install as it can be installed to a USB or hard drive alongside a current operating system or as a complete replacement. Vinux can also run from a live $\mathrm{CD}$ or Live USB without making any changes to a current operating system. It also has a large friendly community to discuss issues. Its interface is just like Ubuntu's interface. Vinux installs English by default but provides support for installation and configuration of other languages. It provides the promised set of functionalities, and continuous updates are made by its developers.

\section{Emacspeak}

Emacspeak[26] is subsystem of Emacs which is an interface of Debian version of Linux operating system. Emacspeak provides a complete speaking desktop by speech enabling in all of Emacs. It is open source software which is very helpful for those people who are blind and they rely on their hearing sense. With Emacspeak they do anything that a normal person can do. So we can say that Emacspeak is not just a screen reader it is a complete user environment with built in speech recognition. Emacspeak has more context specific information about what is speaking than its relative screen readers.

Emacspeak consists of a core speech system that provides speech and audio services to the rest of the Emacspeak desktop; application-specific extensions provide contextspecific spoken feedback using these services. It has built in speech notifications like when a person is reading a file or deleting a file, etc. It has user custom dictionary in which a user can save its own words. It supports multiple languages.

It is compatible with Linux operating system. Additional requirements are Linux-compatible sound card, a hardware or software speech synthesizer and Emacs. It is open source software so it is free of cost.
Table 3 compares Linux based assistive technology products on set of parameters that have already been defined. The criterion for these parameters is same as defined for Microsoft Windows based assistive technology products.

Table 3: Parameterized comparison of Linux based Assistive technology products

\begin{tabular}{|l|l|l|l|}
\hline Parameters & Orca & Vinux & Emacspeek \\
\hline Performance & High & High & High \\
\hline Usefulness & High & High & High \\
\hline User friendly & Medium & Medium & Medium \\
\hline $\begin{array}{l}\text { Local language } \\
\text { support }\end{array}$ & Yes & Yes & Yes \\
\hline Functionality & High & High & High \\
\hline Portability & Yes & Yes & Yes \\
\hline Cost & Free & Free & Free \\
\hline
\end{tabular}

\section{SHORTCOMINGS AND RECOMMENDATIONS}

The significant constraint for blind persons on the use of computers is lack of sight and for this they completely rely on the sense of hearing. So, for getting just the required piece of information, the blind person has to listen to a long speech. They have to go through entire document or article to find either it is the required one or not. A blind user has to wait to listen to get desired heading, even jumping from heading to heading, due to slow speed of screen reader. Even though turning the speech settings to fast will still not be as fast as for a normal user.

It's high time to fix the above limitations, developers should consider of using principle such as from less speech to generate maximum output. This will increase utilization of screen readers. If a user somehow is unable to grab or misses some of the speech, user will have to go through again, thus requiring the whole process to repeat them. Importantly user must have control on whatever is being said: sighted persons are out of bound to look wherever and whatever in the documents. Such kind of manageable focus should also be provided to the visually impaired persons. Means such users must have control to navigate through the document, stop, go back, skip items, repeat and explore the available text.

Computer usage is not that difficult for deaf persons as in the case of blind persons as most of computer actions require visual contact with it. So, smaller number of adaptations is required. Most of the time, the computer tasks can be easily completed without hearing aid such as sending an e-mail, editing a document, browsing the web pages, computing the mathematical equations however certain actions do involve sound such as creating an alert or indication of an error. For example, we hear a sound on receiving an e-mail or hear an annoying sound on occurrence of an error. Therefore, the messages should be prompt on the screen to the user without using the sound. On the other hand Speech/Text to Video Sign-Language translators needs to be more effective. So that deaf persons can easily communicate in educational, workplace, and public access venues in the absence of interpreters.

Loss of physical mobility makes maximal participation in performing desired activities more difficult and in the worst case fully prevents participation. It is a challenge to develop systems for such people because they may interact with computer using their vision, tongue (speech), hand, foot, or 
head movement. Sometimes the input given by user is missed or not traced due to the inefficiency of present assistive technology gadgets. The assistive technology tools present for mobility impaired people need to be more effective, responsive, easy to use and mature. Because mobility impaired person may also suffering from some other issues too i.e. loss of balance, weakness, fatigue, cognitive impairment, fear of falling, spasticity, tremor, and visual impairment.

The Linux based assistive technology provides high functionalities and is free of cost. But, these products lack user manual or products support documents. These products have friendly support groups but the deficiency of user manuals can't be fulfilled by them. Another issue is complex GUI support. So detailed user manuals and small GUI changing are required to bring them at the level of other competitive products. Along with all these adaptations, it is highly recommended that the prices of assistive technology products must be lessen because most of the windows based assistive technologies are very expensive.

\section{CONCLUSION AND FUTURE WORK}

With the rapid change in technology, it is important for researchers and developers to keep up with new technology that could assist disabled persons. Continued research and development in the area of assistive technology is essential with the explosion of new technologies. According to the review conducted in this paper, an ideal set of systems is required which have the properties to learn from the routine tasks of the disabled person as well as able to understand the person for whom it is designed. It's the only way that it will be able to overcome the areas of deficiencies. In addition the cost of such system wouldn't be high as it is not designed for normal persons. So a disabled person may not be able to afford to buy such a system. This survey paper shows that a lot of work is done but still much more is required to understand and accommodate these people in their cause of distress.

In this paper, analysis is based on the information provided by the vendors and users online. The recommendations are also based on the reviewer's personal analysis and user's reviews about product available online. In the future, the survey would be conducted to analyze the hurdles faced by disabled persons while using assistive technology products.

\section{REFERENCES}

[1] Media centre disability and health fact sheet, World health organization, [online] 2014, http://www.who.int/mediacentre/ factsheets/fs352/en/ (Accessed: $15^{\text {th }}$ March 2015).

[2] Children with disabilities, UNICEF, state of the world's children, [online] 2006, www.unicef.org/sowc06/profiles/disabilities.php, (Accessed: $15^{\text {th }}$ March 2015).

[3] Access Technology Training-What is accessed technology? Royal National Institutes of blinds, [online] http://www.rnib.org.uk/

professionals/solutionsforbusiness/trainingandconferences/techn ology/Pages/access_technology_training.aspx (Accessed: $15^{\text {th }}$ March 2015).

[4] Ronald J. Seiler, "Assistive Technology for Individuals with Cognitive Impairments, The Idaho Assistive Technology
Project", Center on Disabilities and Human Development, University of Idaho, Moscow, December 2007.

[5] Behrmann, M. M., Jones, J. K., \& Wilds, M. L, "Technology intervention for very young children with disabilities", Infants and Young Children, vol. 1, pp. 66-77, April. 1989.

[6] Y. Saleem , A. Ahmad, O. B. Naeem, M. F. Hayat, S. M. Afzaal, M. Amjad and M. Saleem, "Windows Interface For Disabled Person", Pakistan Journal of Science, March 2014.

[7] Accessibility Technology Products for Windows, Microsoft Accessibility, [online], http://www.microsoft.com/enable/at/ matvplist.aspx (Accessed: $17^{\text {th }}$ March 2015).

[8] Sandra Alper, Sahoby Raharinirina, " Assistive Technology for Individuals with Disabilities: A Review and Synthesis of the Literature ", Journal of Special Education Technology, March 2006.

[9] James A. Lenker M.S, A.T.P. and Victor L, "A Review of conceptual Models for Assistive Technology Outcomes Reseach and Practice", Assistive Technology: The official journal of RESNA, vol. 15, pp. 1-15, 2003.

[10] Hoppestad BS., " Inadequacies in computer access using assistive technology devices in profoundly disabled individuals: an overview of the current literature", Disability and Rehabilitation Assistive Technology, vol. 2, pp. 189-199, July 2007.

[11] Charlotte A. Mull, Patricia L. Sitlington "The Role of the technology in the transition to postsecondary education of students with disabilities: A review of literature ", Technology and Health Care, November 2009.

[12] Head mouse extreme wireless head controlled mouse, Origin instruments, [online], http://www.orin.com/access/headmouse/ (Accessed: 21 ${ }^{\text {st }}$ March 2015).

[13] Foot time mouse foot controlled hands free mouse, Fentek industries, [online], http://www.fentekind.com/footime.htm\#VR14xLrBzGo (Accessed: 21 ${ }^{\text {st }}$ March 2015).

[14] SoftType 4.2 English on-screen keyboard, Boundless assistive technology, [online], http://www.boundlessat.com/Mobility/Onscreen-KeyboardsMice/SoftType-4-2-English-on-screen-Keyboard (Accessed: $21^{\text {st }}$ March 2015).

[15] Icommunicator the finest software ever developed for people who are deaf or hard of hearing, [online], http://www.icommunicator .com/productinfo/index.html (Accessed: $21^{\text {st }}$ March 2015).

[16] JAWS for windows, Nanopak. inc, [online], http://www.nanopac.com/jaws.htm (Accessed: 21 ${ }^{\text {st }}$ March 2015).

[17] Clarity smart for seniors, JV35, [online], shop.clarityproducts. com/products/clarity/jv35-amplifiedtelephone-with-talk-back-numbers/ (Accessed: 21 ${ }^{\text {st }}$ March 2015).

[18] NV access home of the free NVDA screen reader, [online], http://www.nvaccess.org/ (Accessed: 21 ${ }^{\text {st }}$ March 2015).

[19] Blindness solutions: open book and pearl, Freedom scientific, http://www.freedomscientific.com/Products/Blindness/ OpenBookAndPearl (Accessed: $21^{\text {st }}$ March 2015).

[20] Darren Burton and John Lilly, “AFB NonVisual Desktop Access and Thunder: A Comparison of Two Free Screen Readers", AFB Access World Magazine [online], vol. 12, number. $8, \quad$ August. 2011. www.afb.org/afbpress/pub.asp?DocID=aw120803 (Accessed: 5th May 2015).

[21] Assistive Technology: JAWS Screen Reader, [online], http://www2.lib.udel.edu/atc/jaws.pdf (Accessed: 5th May 2015). 
[22] NVDA - a free and open source screen reader for

Windows, [online],

http://www.paciellogroup.com/blog/2008/01/nvda-a-free-andopen-source-screen-reader-for-windows/ (Accessed: 5th May 2015).

[23] Adnan Rawashdeh and Baseem Matalkah, "A New Software Quality Model for Evaluating COTS Components", Journal of Computer Science, 2006.

[24] F. Reena Sharma and S. Geetanjali Wasson, " Speech Recognition and Synthesis Tool: Assistive Technology for Physically Disabled Persons", International Journal of Computer Science and Telecommunications, 2012.

[25] Window-Eyes, [online], http://screen-reader-softwarereview.toptenreviews.com/window-eyes-review.html, (Accessed $5^{\text {th }}$ may 2015).

[26] Emacspeak--The Complete Audio Desktop, [online], http://emacspeak.sourceforge.net/, (Accessed $27^{\text {th }}$ may 2015).

[27] Vinux Project, [online], http://vinuxproject.org/, (Accessed $27^{\text {th }}$ may 2015).

[online], https://wiki.gnome.org/action/show/Projects/Orca? action=show\&redirect=Orca, $\left(\right.$ Accessed $27^{\text {th }}$ may 2015$)$.

[29]Linux Accessibility HOWTO, [online], http://www.tldp.org/HOWTO/AccessibilityHOWTO/linuxos.html (Accessed $27^{\text {th }}$ may 2015). 